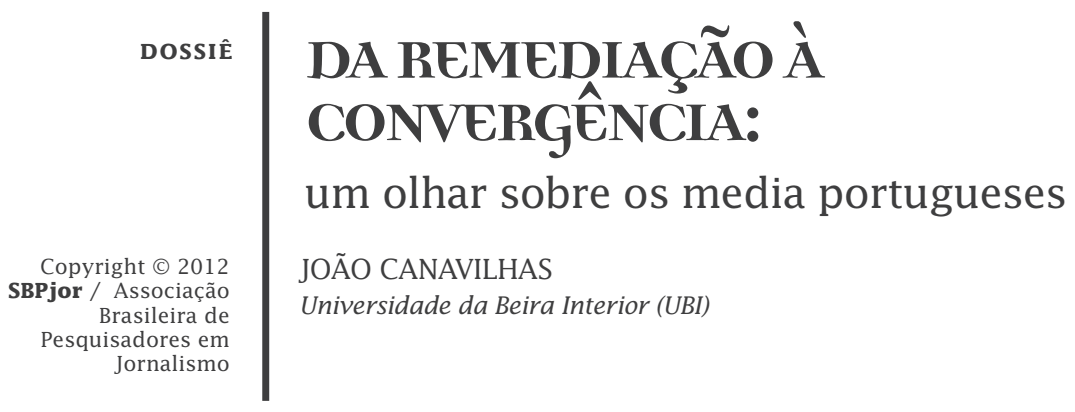

RESUMO - A digitalização no setor do jornalismo e a emergência da Internet transformaram a convergência mediática num novo campo de investigação. Numa primeira fase, os investigadores concentraram-se nos processos de convergência dentro das, nomeadamente nas questões relacionadas com a reorganização empresarial (KETTERER et al.,2004; QUINN, 2005) e com as rotinas profissionais dos jornalistas (SALAVERRÍA, 2003; MASIP; MICÓ, 2009; VERWEIJ, 2009). Mas cedo se percebeu que a convergência influenciava uma área mais vasta. A migração dos meios tradicionais para a Internet e a rápida evolução tecnológica mostraram que a convergência era um fenômeno transversal que ocorria também no campo dos conteúdos (CÁDIMA, 1999; QUINN; FILAK, 2005), na distribuição de notícias (BRUNS, 2003; CABRERA, 2010) e no consumo de informação (BOWMAN; WILLIS, 2003; JENKINS, 2006; SUNDET; YTREBERG, 2009). Porque acreditamos que a convergência de conteúdos implica necessariamente uma convergência nos processos a montante, neste trabalho debruçamo-nos exclusivamente sobre o resultado do processo noticioso: os conteúdos jornalísticos. Com base na realidade portuguesa procuramos diferenciar os conceitos de convergência e remediação (BOLTER; GRUSIN, 1999), identificando marcas características dos dois processos.

Palavras-chave: Convergência. Remediação. Jornalismo. Novas mídias.

\title{
FROM REMEDIATION TO CONVERGENCE looking at the Portuguese media
}

\begin{abstract}
The digitalization in the journalism sector and the emergence of the Internet have transformed media convergence into a new field of research. In a first stage, researchers were centered on convergence processes in the newsroom, namely in questions related with entrepreneurial reorganization (KETTERER et al., 2004; QUINN, 2005) and journalists' professional routines (SALAVERRÍA, 2003; MASIP; MICÓ, 2009; VERWEIJ, 2009). However, it was soon understood that convergence influences a larger area: the migration of traditional media to the Internet and the rapid technological evolution showed that convergence was a transversal phenomenon that also happened in the contents field (CÁDIMA, 1999; QUINN; FILAK, 2005), news distribution (BRUNS, 2003; CABRERA, 2010) and information consumption (BOWMAN; WILLIS, 2003; JENKINS, 2006; SUNDET; ESPEN 2009). The present work is centered on the study of contents convergence, attempting to produce an accurate portrait of the Portuguese media situation.
\end{abstract}

Keywords: Convergence. Remediation. Journalism. New media. 
Welcome to convergence culture, where old and new media collide, where grassroots and corporate media intersect, where the power of the media producer and the power of the media consumer interact in unpredictable ways (JENKINS, 2006, p.2)

\title{
CONVERGÊNCIA VS REMEDIAÇÃO
}

Apesar da multiplicidade de estudos sobre o fenômeno da convergência, não existe uma definição que reúna consenso entre os investigadores da área (SALAVERRÍA; GARCIA AVILÉS; MASIP, 2010). Dentro das definições que procuram enquadrar o fenômeno de uma forma mais ampla, Jenkins (2006, p. 2) define convergência como "the flow of content across multiple media platforms, the cooperation between multiple media industries, and the migratory behaviour of media audiences who will go almost anywhere in search the kind of entertainment they want". Nota-se aqui uma preocupação com os conteúdos e as organizações, mas também com o papel das audiências, uma variável muitas vezes ignorada pelos investigadores. Na mesma linha, Machado e Teixeira (2010, p. 13) recorrem a vários autores para definir convergência como

\begin{abstract}
um conceito complexo e polifônico utilizado para definir a produção integrada e contínua de informações por uma mesma ou por distintas equipas para múltiplas plataformas e com formatos e linguagens próprios de cada uma, em uma organização composta por meios anteriormente atuando como processos de produção autônomos .
\end{abstract}

Também neste caso podemos constatar uma tentativa de integrar as várias facetas da convergência, procurando uma definição chapéu capaz de abraçar todos os campos da convergência. Esta é, aliás, uma tendência na área, sendo muitos os investigadores que procuram definir a convergência como um processo multifatorial: veja-se o caso de Dupagne \& Garrison (2006), que defendem a análise da convergência nos campos técnico, econômico e legislativo. Jenkins (2006) defende igualmente que a convergência seja analisada em três grandes setores, embora diferentes dos anteriores: distribuição de informação para multiplataformas, cooperação entre diferentes empresas e migração e comportamento das audiências. Por fim, Salaverría (2010) propõe que a análise se faça em quatro campos: convergências de conteúdos, tecnológica, empresarial e profissional.

Das três propostas referidas, a de Salaverría é a que mais nos interessa porque destaca claramente aquele que é o nosso objeto de estudo: os conteúdos: Consideramos que este é o elemento fundamental na identificação de um processo de convergência porque a existência de conteúdos com linguagens específicas adaptadas ao meio implica 
necessariamente que nos campos situados a montante se tenham verificado as restantes convergências. Tal como referem Machado e Teixeira (2010), a convergência envolve uma produção integrada de conteúdos para diferentes plataformas, utilizando para isso linguagens próprias. Na mesma linha, Boczkowski (2006) refere que convergência pode ser o fornecimento de um conteúdo multimediático que junta as características de conteúdos antes distribuídos por diferentes meios, o que remete para McLuhan (1964) quando diz que o conteúdo de qualquer meio é sempre outro meio. Estes autores coincidem assim num ponto fundamental: só se pode falar de convergência quando o produto final é um conteúdo com características únicas.

Esta clarificação permite um afastamento em relação a autores que associam o conceito de convergência a uma simples distribuição multiplataforma (QUINN, 2005; JENKINS, 2006), sem referirem explicitamente a necessária adaptação dos conteúdos às características dos dispositivos de recepção. E é aqui que reside a grande diferença entre os processos de convergência e de remediação.

Por remediação (remediation) entende-se o processo de renovação de velhos conteúdos efetuado pelos novos meios. Ou seja, os novos meios de comunicação renovam (refashion) os conteúdos dos anteriores, permanecendo desta forma uma ligação entre novos e velhos meios (BOLTER; GRUSIN, 1999). Este processo pode ocorrer em diferentes níveis: no caso dos meios digitais, o nível pode variar entre uma melhoria discreta do meio antecessor, mantendo algumas das suas características, até remediações mais profundas, em que o novo meio digital tenta absorver completamente o anterior. Bolter e Grusin (1999) sublinham que o velho meio está sempre presente porque, de uma forma mais ou menos visível, o novo meio depende sempre do anterior, criandose até uma relação semântica entre os dois: "all current media function as remediators and that remediation offer us a means of interpreting the works of earlier media as well' (BOLTER; GRUSIN, 1999, p. 55).

No campo dos conteúdos, os autores consideram que "convergência" e "remediação" são semelhantes, dizendo mesmo que "convergence is remediation under another name" (BOLTER; GRUSIN, 1999, p. 224). Discordamos desta posição: é verdade que em ambos os casos falamos de conteúdos resultantes de outros usados pelos meios anteriores, mas há uma diferença importante entre os dois conceitos: enquanto a convergência implica necessariamente uma nova linguagem que integre os conteúdos anteriores, a remediação pode ser uma acumulação de conteúdos de diferentes origens distribuídos 
numa mesma plataforma. Nesse sentido, a convergência é sempre uma remediação, mas nem todas as remediações podem ser consideradas uma convergência porque esta última implica integração e não uma mera acumulação de conteúdos.

\section{Meios tradicionais: a remediação como etapa da convergência digital}

A história dos mass media mostra que cada novo meio passa por um período de indefinição até estabilizar um conjunto de características próprias. Isto significa que os novos meios começam por misturar os conteúdos dos seus antecessores (remediação) até estabilizarem uma linguagem própria (convergência).

A título de exemplo veja-se o caso da rádio: o som, elemento natural deste meio, pode ser transmitido em forma de voz, música ou ruído. A voz (comunicação verbal oral) é aqui uma remediação do texto (comunicação verbal escrita) característico da imprensa. Nos primeiros anos da rádio, a colocação de voz dos animadores e jornalistas evidenciava que os profissionais estavam a ler textos num registo próximo da leitura de um jornal. Posteriormente a rádio tentou aproximar-se da cultura oral e por isso procurou um registo "entre la lectura de textos y la improvisación oral' (GONZÁLEZ, 1996, p. 49). Como diz Meneses (2003, p. 101), "há dois registos diferentes e quase contraditórios na rádio: o lido e o falado. O segundo é desejado, o primeiro é a realidade". Este período de adaptação do texto escrito a um meio oral exemplifica o processo de remediação característico dos media. No caso da rádio, a convergência só se materializou quando à voz se juntaram a música, o ruído e o silêncio, criando aquilo a que Balsebre (apud SOENGAS, 2003) chama as "imagens auditivas", ou seja, o poder que o som tem para gerar imagens mentais, transmitir sensações e criar ambientes graças à sua combinação com a voz num registo sonoro adaptado ao meio. Não se tratou simplesmente de juntar diferentes tipos de sons, mas de encontrar um conjunto de regras que estão na base da construção de uma notícia radiofônica.

No caso da televisão, a marca distintiva é a imagem sonorizada. Quando é fixa, a imagem é uma remediação da fotografia, embora neste caso adaptada ao formato televisivo (4/3 ou 16/9). Já o registo áudio que acompanha as imagens é uma remediação do som radiofônico, embora com algumas diferenças: 
y ser coherente con las mismas para que no se produzca una falta de raccord, pero, al mismo tiempo, debe añadir datos que no se aprecian en los planos y facilitar detalles que los espectadores no descubren con la simple contemplación (SOENGAS, 2003, 10).

Neste caso já é possível identificar um estado inicial de convergência devido à introdução do raccord, ou seja, da obrigatoriedade de existir uma sequência lógica entre planos. Estamos perante um caso em que o novo meio junta conteúdos antes distribuídos por outros meios, mas fá-lo por integração, dando assim origem a um produto diferenciado.

Para além de passarem por este processo de remediação, que evoluiu até à convergência atual, é igualmente possível identificar exemplos de remediação inversa, ou seja, processos em que o meio antecedente procura incorporar características do meio emergente. O formato antena aberta, característico da rádio, é um exemplo de remediação inversa na televisão: estes programas, em que os ouvintes telefonam para opinar sobre determinado tema, são hoje uma presença regular na programação dos canais temáticos de informação. Nestes casos, a imagem é absolutamente desnecessária porque as intervenções ocorrem via telefone e os apresentadores pouco mais fazem do que gerir o tempo atribuído aos telespectadores previamente selecionados. Outro exemplo interessante de remediação inversa acontece nos jornais: os espaços de perguntas e respostas para definição de determinados conceitos são uma adaptação das FAQ's (Frequently Asked Questions) características da Web.

O aparecimento da Internet tornou mais visíveis os fenômenos de remediação - direta ou inversa - e atualmente quase todos os meios de comunicação tradicionais criaram versões on-line ou adaptaram algumas características da Web. No caso português, jornais, rádios e televisões continuam a evoluir num percurso de remediação, havendo poucos exemplos de convergência de conteúdos.

\subsection{Imprensa}

Os jornais foram o primeiro meio a criar versões na Web: em apenas cinco anos, a maioria das publicações de cariz jornalístico lançou versões on-line procurando acompanhar o crescente interesse dos utilizadores pelo novo meio (ALVES, 2001). O fato da primeira vaga de meios a chegar à Web ser majoritariamente composta por jornais fez com que o texto e as fotos fossem os conteúdos dominantes na primeira geração de jornalismo na Web. No caso português, os 
primeiros a chegar foram o Jornal de Notícias e o Público, os dois em 1995. O primeiro jornal exclusivamente on-line, o Setúbal na Rede, só nasceria três anos mais tarde, em 1998. Esta vanguarda assumida pelos jornais ficou a dever-se ao fato de os programas de edição eletrônica usados nas redações permitirem exportar os conteúdos em HTML, o que facilitou a migração dos jornais para a Web sem necessidade de grandes investimentos em recursos humanos ou equipamentos. Nesta fase, as publicações limitavam-se a transpor os conteúdos do papel para a Web (shovelware) tentando valorizar o valor da marca off-line (KRUEGER; VAN DER BEEK; SWATMAN, 2004), única fonte de receitas. Desde então os meios tradicionais com versões na Web iniciaram um percurso de remediações a caminho da desejável convergência, situação que num futuro próximo dificultará a identificação do meio que Ihe deu origem.

Tendo sido o primeiro meio a chegar à Internet, a imprensa escrita tem também o mais longo percurso evolutivo. Depois da fase de shovelware, os jornais procuraram integrar algumas das características da Web nas suas versões on-line. De uma forma progressiva, hipertextualidade, multimedialidade, interatividade, instantaneidade, personalização, memória e ubiquidade (BARDOEL; DEUZE, 2001; DÍAZ NOCI, 2001; HALL, 2001; MACHADO; PALACIOS, 2003) têm vindo a ser integradas nas versões on-line, porém a um ritmo mais lento do que seria desejável.

Se as edições on-line da imprensa escrita estão longe de oferecerem conteúdos convergentes, nas suas versões tradicionais oferecem algumas experiências interessantes de remediação inversa: um bom exemplo deste processo é a utilização de imagens em movimento. Em outubro de 2008, a Esquire assinalou o seu $75^{\circ}$ aniversário publicando uma edição especial com a primeira capa do mundo a utilizar e-ink $k^{1}$ (tinta eletrônica): esta foi a primeira distribuição em massa de uma publicação com imagens em movimento. Mais recentemente, a imprensa passou a utilizar a realidade aumentada, uma outra possibilidade de "integrar" vídeo nas edições impressas. Apontando uma webcam a códigos impressos na revista, o leitor pode assistir a um conjunto de vídeos na tela do computador ou do telefone celular.

\subsubsection{A imprensa em Portugal}

No caso português, as edições on-line dos jornais ganharam alguma autonomia de conteúdos, mas a convergência está longe de se verificar.

A multimedialidade continua a fazer-se por acumulação e 
não por integração: os vídeos apresentados - sobretudo notícias e entrevistas - são elementos autônomos que repetem a informação textual. A linguagem audiovisual utilizada é idêntica à da televisão, pelo que estamos perante uma mera uma remediação.

A hipertextualidade resume-se à oferta de hiperligações para notícias relacionadas existentes no arquivo (memória), sendo raros os casos em que esses links são inseridos nos textos: o mais habitual é situarem-se num menu lateral ou no final da notícia.

A participação dos leitores, forma de interatividade mais usada, é igualmente discreta: inquéritos/votações e a possibilidade de comentar a notícia são as alternativas oferecidas, porém esses comentários nunca são aproveitados para enriquecer a notícia, mesmo que acrescentem informações pertinentes.

Entre os novos formatos, outra forma de identificar um processo de convergência, apenas as galerias fotográficas e as infografias lineares têm alguma utilização. Porém são formatos de baixa complexidade e muito semelhantes a outros já utilizados na televisão e na imprensa, respectivamente. Entre as publicações portuguesas, os diários Público ${ }^{2}$, e Jornal de Notícias ${ }^{3}$, em conjunto com o semanário Expresso ${ }^{4}$, são os jornais que melhor exploram as potencialidades da Internet, mas a diferenciação dos conteúdos em relação às versões escrita é diminuta. No campo dos jornais digitais nativos o panorama é ainda mais desolador: os títulos são escassos e a falta de recursos humanos deixa pouco espaço para que os jornais tenham sequer uma agenda própria.

No campo da remediação inversa existem algumas experiências nos jornais e revistas portuguesas. A adaptação das FAQs é utilizada pelo jornal Público (P\&R - Perguntas e Respostas), que nesta coluna clarifica questões relacionadas com a notícia. Também o jornal $I^{5}$ utiliza na sua versão impressa uma espécie de sucedâneo de hipertexto, sublinhando determinadas palavras e ligando-as através de uma linha a mais informação ou a URLs.

Um dos casos em que podemos falar de convergência acontece na revista Sábado, que desde julho de 2010 tem utilizado a Realidade Aumentada. A revista imprime nas suas páginas códigos gráficos que acedendo a um site criado para o efeito ${ }^{6}$ permitem ao leitor ver no seu computador ou smartphone um conjunto de vídeos complementares ao texto. Esta utilização da Realidade Aumentada é um bom exemplo de convergência porque os conteúdos apresentados fazem parte integrante da notícia e complementam a informação escrita. 


\subsection{A Rádio}

No caso da rádio tradicional, a mudança mais evidente ocorreu ao nível da interatividade: o característico contato telefônico com os ouvintes é agora complementado com opiniões chegadas ao estúdio através das redes sociais. Surgiram igualmente alguns conteúdos inspirados em aplicações da web 2.0, abrindo mais espaço à voz dos ouvintes.

Nas versões Web, a rádio teve um início semelhante aos dos jornais, usando sobretudo texto e fotos. Neste caso não há uma relação com os conteúdos do meio original, mas sim uma utilização de conteúdos adaptados às ligações dial-up características dos primeiros tempos. Por isso mesmo, as versões web das rádios sempre foram muito diferentes da sua versão tradicional: "na Internet, a rádio afasta-se do seu conceito original e, no website, pode apresentar serviços distintos da emissão radiofônica, estabelecendo uma nova estrutura, mais rica e variada que concorre diretamente com o formato tradicional da rádio" (CORDEIRO, 2004 , p. 2). Esta visão de "rádio concorrente" permite vislumbrar um processo de remediação mais avançado, a ponto de se poder falar mesmo em convergência.

\subsubsection{A rádio em Portugal}

Na emissão tradicional da rádio, a grande mudança foi a entrada das redes sociais nos programas de animação. Este fato melhorou o espaço de intervenção dos ouvintes, que passaram a ter ferramentas de interação mais eficazes do que os tradicionais telefonemas. Surgiram igualmente alguns programas influenciados pela Web (O meu blogue dava um programa de rádio $^{7}$ - Rádio Comercial): neste exemplo de remediação inversa, a realizadora do programa procurava sonorizar o espírito dos blogues, lendo alguns posts publicados nestes espaços.

$\mathrm{Na}$ versão $W e b$, as rádios portuguesas começaram pela referida aproximação ao modelo da imprensa, mas em 1996 a TSF ${ }^{8}$ já oferecia a emissão sonora na sua página. Três anos depois, o site desta estação de rádio apresentava notícias hipertextuais e podcasts, dois formatos próprios do novo meio. Embora o podcast se tenha generalizado entre as rádios portuguesas, a hipertextualidade não evoluiu, havendo sobretudo uma aposta na multimedialidade. Atualmente, a generalidade das rádios, incluindo as locais, oferece conteúdos semelhantes aos das televisões, nomeadamente notícias e entrevistas em vídeo. O melhor exemplo desta realidade ${ }^{9}$ é a Rádio Renascença por ter uma vasta oferta de vídeo com notícias, reportagens e até serviços informativos completos ( $\mathrm{V}+$ ). 
Este modelo de multimedialidade por acumulação continua a ser uma remediação dos conteúdos televisivos, uma vez que não se vislumbra qualquer marca de adaptação ao meio, tal como acontece nas televisões.

Mas a rádio na Web portuguesa também apresenta marcas de convergência. No caso da TSF nota-se a utilização do som como complemento ao texto, substituindo as citações por ficheiros áudio (Rms). Infelizmente este formato ainda é pouco utilizado: o mais habitual é a oferta da notícia integral em texto e em som, sendo neste segundo caso a versão transmitida na emissão hertziana.

Na Rádio Renascença, a convergência é visível na existência de alguns gêneros nativos da Web, como infografias multimédia ${ }^{10}$. O exemplo desta rádio deve ser destacado porque tem uma oferta multimédia de grande qualidade e variedade, embora pertença a um grupo de comunicação que não tem jornais nem canais de televisão nacionais.

\subsection{A Televisão}

$\mathrm{Na}$ televisão hertziana, as marcas da Internet são muito semelhantes às registadas na rádio. No campo da interatividade com os telespectadores, a utilização do e-mail e das redes sociais é hoje prática corrente em muitos programas de entretenimento e até de informação. Com a televisão digital abriram-se novas possibilidades no campo da interação, podendo os telespectadores personalizar as grades de programação e, nalgumas transmissões em direto, escolher os planos, selecionando a câmara melhor posicionada no terreno.

No que concerne às versões on-line, os primeiros sites das televisões mais pareciam brochuras eletrônicas da estação, exibindo a programação e alguma informação sobre os conteúdos. No início do novo século, as televisões começaram a incluir notícias, mas, tal como os restantes meios on-line, em formato textual devido a questões de ordem técnica relacionadas com a largura de banda. A generalização da banda larga e os novos formatos de compressão de vídeo tornaram o processo mais rápido, permitindo às televisões emitir em streaming e disponibilizar programas em vodcast. Entre os meios de comunicação tradicionais, a televisão é aquele que menos alterou a sua natureza nas versões web, tendo neste meio uma espécie de canal não linear onde o utilizador pode construir uma programação à sua medida.

\subsubsection{A televisão em Portugal}

A remediação inversa na televisão portuguesa observase sobretudo na crescente utilização das redes sociais: nalguns 
programas de informação existe um jornalista/animador em estúdio para introduzir os comentários e perguntas dos telespectadores no debate (Pontapé de Saída na RTPN; 5 para a meia-noite - RTP2). O episódio que melhor ilustra a interação com recurso à Web 2.0 é a entrega de um hambúrguer a Alberta Marques Fernandes, jornalista da RTP. Enquanto preparava o telejornal, e no âmbito de uma discussão sobre fast-food que decorria no Twitter, a jornalista escreveu no seu mural que lhe apetecia um hambúrguer. Um dos internautas decidiu responder ao pedido e a partir desse momento iniciou a sua missão, fotografando e publicando cada passo até chegar à RTP para entregar o hambúrguer. Enquanto isso, a comunidade mantinha-se em contato com a jornalista que estava prestes a iniciar a apresentação do telejornal. O objetivo foi cumprido e tudo terminou com um sinal em direto para confirmar a receção do pedido ${ }^{11}$. Embora se trate de um episódio pitoresco e sem influência nos conteúdos, este caso mostra bem até o potencial que as redes sociais podem ter na televisão tradicional.

Ainda no campo da emissão tradicional, saliente-se a crescente utilização de fotos e vídeos enviados pelos telespectadores. Esta situação já acontecia antes da digitalização (Isto só Visto - RTP), mas com a vulgarização das câmaras digitais e dos smartphones passou a ser uma presença habitual também na informação, sobretudo em casos de acidentes ou fenômenos da natureza. Os serviços informativos de algumas estações incluem mesmo rubricas exclusivamente compostas por fotos enviadas pelos telespectadores (verão SIC).

No que concerne às versões on-line das televisões portuguesas, só a partir de 2002 a multimedialidade passou a ser uma oferta regular nos sites televisivos, porém não houve qualquer preocupação em adaptar os vídeos ao meio, uma situação que perdura até aos dias de hoje. As notícias exibidas na televisão tradicional são comprimidas e reduzidas para um formato menor sem que se verifique qualquer preocupação relacionada com o tipo de plano a ser utilizado num formato mais reduzido. Trata-se de uma mera remediação à qual é acrescentada uma das características do jornalismo na Web - a memória - pois as notícias aparecem sempre associadas a outras sobre o mesmo tema.

A interatividade e a personalização também estão presentes nas versões web das televisões, no primeiro caso com a possibilidade de comentar/partilhar e no segundo com aplicações (O Meu Telejornal ${ }^{12}$, - RTP) que permitem ao utilizador construir um telejornal a seu gosto.

Uma nota final para referir que o site da $\mathrm{TV}^{13}$ faz uma utilização 
esporádica da hipertextualidade oferecendo ligações a notícias de outros sites especializados pertencentes ao mesmo grupo de comunicação.

\section{Objetivo: Convergência}

Ao longo da história, os novos meios começaram por remediar os seus antecessores para em seguida adquirirem a sua própria linguagem. Paralelamente verificou-se um processo de remediação inversa em que o meio antecessor procurou sempre agregar algumas das características do novo meio, tentando desta forma resistir ao natural desgaste de audiências provocado pela entrada de um novo meio no ecossistema mediático.

Com a emergência da Internet e a possibilidade de todos os meios migrarem para este novo espaço, criou-se a ideia de que se verificaria uma convergência de conteúdos e aparecimento de uma nova linguagem. Em rigor, o que se tem verificado em Portugal é aquilo a que Salaverría (2010, p. 39) chama "confluencia de contenidos", ou seja, os grupos que integram jornais, rádios e/ou televisões transportam para as versões digitais os conteúdos de todos os seus meios criando uma sensação de multimedialidade que não é mais do que uma oferta tripla da mesma informação.

Usando a classificação de Salaverría (2010), diríamos que a "convergência de conteúdos" (multimedialidade) está muito atrasada em relação à maturidade já atingida pelas convergências tecnológica (multiplataforma), empresarial (concentração) e profissional (polivalência). A atual multimedialidade por acumulação é uma mera remediação e não pode ser vista como um fenômeno de convergência de conteúdos: defendemos que isso apenas acontece quando é utilizada uma linguagem própria com características únicas e diferenciadoras em relação aos restantes meios.

Em termos de conteúdos jornalísticos, e no que se refere ao campo do gênero notícia, a convergência implica a implementação de um conjunto de alterações ao nível da arquitetura da notícia, bem como a existência de regras para uma adaptação dos vários tipos de conteúdos a diferentes situações. Canavilhas (2007) propõe uma arquitetura (Pirâmide Deitada) e uma gramática multimédia que procuram indicar como, quando e onde devem ser utilizados links, vídeos, sons, fotos e infografias. A proposta pretende ser um ponto de partida para a estabilização de um conjunto de regras que permita ao jornalismo passar da fase de remediação para a de conteúdos convergentes, explorando eficazmente as potencialidades da Web.

A hipertextualidade, entendida como a possibilidade de ligar 
blocos de informação com links, tem sido mais usada em menus e em ligações a notícias relacionadas, o que limita o seu potencial. Enquanto unidades narrativas, os links têm uma função semântica ligada à ampliação da informação, e uma segunda função de apoio à navegação, indicando pistas de leitura. Como se viu, nenhum meio de comunicação português usa esta característica com regularidade, funcionando apenas para ligar as notícias a assuntos relacionados ou a notícias anteriores (memória).

A multimedialidade, definida como a capacidade de uma notícia incluir conteúdos de várias naturezas (SALAVERRÍA, 2005), tem uma forte presença nos jornais on-line, sobretudo no campo do vídeo. $\mathrm{Na}$ maioria dos media portugueses trata-se de uma multimedialidade por acumulação, isto é, as publicações apresentam a mesma notícia nos formatos vídeo e texto, em lugar de existir uma complementaridade entre os dois conteúdos. Esta opção impede a criação de um valor acrescentado que justifique o pagamento pelo acesso aos conteúdos.

No caso da interatividade, as experiências vão pouco além do nível de instrução, em que o utilizador escolhe percursos de leitura clicando em links. Os restantes níveis identificados por Cairo (2008), manipulação (possibilidade de alterar características dos objetos participando em inquéritos que fazem parte da própria notícia) e exploração (integração em ambientes virtuais ou simulação), têm uma utilização residual.

A personalização, uma característica atualmente muito utilizada devido à emergência dos dispositivos móveis como plataformas de acesso às notícias, também é pouco explorada, resumindo-se à possibilidade de sindicar os conteúdos.

Para além da utilização destas potencialidades da Web nas notícias, a convergência implica igualmente o aparecimento de novos gêneros jornalísticos próprios da Web. A infografia multimédia, os serious gamese as reportagens interativas são exemplos de conteúdos nativos convergentes no entanto apenas as infografias multimédia têm uma utilização razoável em Portugal e, ainda assim, com uma qualidade mediana.

As convergências tecnológica, empresarial e profissional "favorecen estas dinâmicas de creciente hibridización de los contenidos y formatos" (SALAVERRÍA, 2010, p. 39), mas para já são poucos os exemplos portugueses de convergência no campo dos conteúdos. Há, por isso, um longo caminho a percorrer desde a atual fase de remediação até à verdadeira convergência de conteúdos caracterizada pela hipermultimedialidade por integração, com vários níveis de interatividade e possibilidade de personalização da informação. 


\section{NOTA}

1 Disponível em: <http://www.esquire.com/the-side/video/e-ink-covervideo>.

2 Disponível em: <www.publico.pt>.

3 Disponível em: <www.jn.pt>.

4 Disponível em: <http://aeiou.expresso.pt/>.

5 Disponível em: <www.ionline.pt>.

6 Disponível em: <http://ra.sabado.pt>.

7 Disponível em: <http://radiocomercial.clix.pt/programas/o_meu_blog.html>.

8 Disponível em: <www.tsf.pt>.

9 Não é feita referência à RDP porque a organização do site dificulta a distinção entre o que é produção da televisão RTP e o que é produzido pela rádio.

10 Disponível em: <http://rr.sapo.pt/Multimedia_Infografia.aspx?fid=106>.

11 Todo o relato Disponível em: <http://caoepulgas.blogspot. com/2009/02/joelysandra-o-twitterheroi.html>.

12 Disponível em: <http://www0.rtp.pt/noticias/index.php?article=20590 $1 \& \mathrm{tm}=8 \&$ layout $=122 \&$ visual $=61>$.

13 Disponível em: <http://www.tvi24.iol.pt/>.

\section{| REFERÊNCIAS}

ALVES, Rosenthal. Reiventando o jornal na Internet. SdP, 32, Vol. 2, 2001. Disponível em: <http://www.saladeprensa.org/art236.htm>. Acesso em: 30 maio 2011.

BARDOEL, Jo; DEUZE Mark. Network Journalism. Converging Competences Of Old and New Media professionals. 2001. Disponível em : <https://scholarworks.iu.edu/dspace/handle/2022/3201>. Acesso em: 15 maio 2011.

BOCZKOWSKI, Pablo. Digitalizar las noticias. Innovación en los diários online. Buenos Aires: Manantial, 2006. 
BOLTER, Jay David; GRUSIN, Richard. Remediation. Understanding New Media. Cambridge: The MIT Press, 1999. 295 p.

BOWMAN, Shayne; WILLIS Chris. We Media: How Audiences are Shaping the Future of News and Information. The Media Center at The American Press, 2003.

BRUNS, Axel. Gatewatching, not gatekeeping: Collaborative online news. Media International Australia Incorporating Culture and Policy: Quarterly Journal of Media Research and Resources, 107, p. 31-44, 2003.

CABRERA, Maria Ángeles. La distribución multiplataforma, objectivo del proceso de convergencia. In LOPEZ, Xosé; PEREIRA, Xosé. Convergencia Digital: Reconfiguración de los Medios de Comunicación en España. Univ. De Santiago de Compostela, 2010. p. 27-40.

CÁDIMA, Francisco Rui. Desafios dos Novos Media. Lisboa: Editorial Notícia, 1999. 125 p.

CAIRO, Alberto. Infografía 2.0: visualización interactiva de información en prensa. Madrid: Alamut, 2008. 128 p.

CANAVILHAS, João. Webnoticia. Propuesta de Modelo Periodístico para la Web. Covilhã: Livros LabCom, 2007. 257 p.

CANAVILHAS, João. El nuevo ecosistema mediático. Revista Index Comunicación, Madrid, n.1, p. 13-24, 2011.

CORDEIRO, Paula. Rádio e Internet: novas perspectivas para um velho meio. BOCC, 2004. Disponível em: <http://www.bocc.ubi.pt/pag/ cordeiro-paula-radio-internet-novas-perspectivas.pdf $>$. Acesso em: 17 maio 2011.

DIÁZ NOCI, Javier . La Escritura Digital: Hipertexto y Construcción del Discurso Informativo. Bilbao: SEUPV, 2001. 233 p.

DUPAGNE, Michel; GARRISON, Bruce. The meaning and influence of convergence. Journalism Studies, volume 7, n. 2, p. 237-255, 2006.

FIDLER, Roger. Mediamorphosis: Understandig New Media.Thousand Oaks: Pine Forge Press, 1997. 302 p.

GONZÁLEZ, Jaime Puig. La Rádio. Barcelona: Rosaljai S.L., 1996. 98 p.

GRANADO, António. Os media portugueses na Internet. Ciberjornalismo, 2002. Disponível em: <http://ciberjornalismo.com/ mediaportugueses.htm>. Acesso em: 19 jun. 2011.

HALL, Jim. Online Journalism: A Critical Primer. London: Pluton Press, 2001. 266 p.

JENKINS, Henry. Convergence Culture: Where old and new media collide. New York: University Press, 2006. 353 p.

KETTERER, Stan; WEIR, Tom; SMETHERS, Steven; BACK, James (2004). Case study shows limited benefits of convergence. Newspaper Research Journal, v. 25, n. 3, p. 52-65, 2004.

KRUEGER, Cornelia; VAN DER BEEK; Kornelia; SWATMAN, Paula. New and Emerging 
Business Models for Online News: A Survey of 10 European Countries. In $17^{\text {th }}$ Bled eCommerce Conference eGlobal, Slovenia, June 21 - 23, 2004.

MACHADO, Elias; TEIXEIRA, Tatiana. Ensino de Jornalismo em tempos de convergência. Rio de Janeiro: e-papers, 2010.

MACHADO, Elias; PALACIOS, Marcos (Eds.) Modelos de Jornalismo Digital. Salvador: Ed. Calandra, 2003. 231 p.

MASIP, Pere; MICÓ, Josep Luis. El periodista polivalent en el marc de la convergencia empresarial. Quaderns del CAC, p. 31-32, 2009.

MCLUHAN, Marshall. Understanding Media: The Extensions of Man. New York: McGraw-Hill, 1964.

MENESES, João Paulo. Tudo o que se passa na TSF... para um Livro de Estilo. Porto: edição Jornal de Notícias, 2003. 325 p.

QUINN, Stephen. Convergence's fundamental question. Journalism Studies, 6 (1), p.29-38, 2005.

QUINN, Stephen; FILAK, Vincent. Writing and Producing Across Media. Oxford: Focal Press, 2005. 277 p.

SALAVERRÍA, Ramón. Convergencia de Medios. Revista Chasqui, n. 81, p. 32-39, 2003.

Redacción Periodística en Internet. Pamplona: EUNSA, 2005. 180 p.

. Estructura de la Convergencia de Medios. In: LOPEZ, Xosé; PEREIRA, Xosé (org). Convergencia Digital: Reconfiguración de los Medios de Comunicación en España. Santiago de Compostela: Servizo de Publicacións e Intercambio Científico, 2010. p. 27-40.

SALAVERRÍA, Ramón; GARCIA AVILÉS, José Alberto; MASIP, Pere. Concepto de Convergencia de Medios. LOPEZ, Xosé; PEREIRA, Xosé (org). Convergencia Digital: Reconfiguración de los Medios de Comunicación en España. Santiago de Compostela: Servizo de Publicacións e Intercambio Científico, 2010. p. 41-64.

SOENGAS, Xosé. Informativos Radiofónicos. Madrid: Ediciones Cátedra, 2003.

SUNDET, Vilde; YTREBERG, Espen. Working Notions of Active Audiences: Further Research on the Active Participant in Convergent Media Industries. Convergence: The International Journal of Research into New Media Technologies, p. 383-390, nov. 15, 2009.

VERWEIJ, Peter. A Case Study of Convergence of Print, Radio, Television and Online Newsrooms at the African Media Matrix in South Africa During the National Arts Festival. Convergence: the International Journal of Research into New Media Technologies, vol. 15, n. 1, p. 75-87, 2009.

João Canavilhas é Doutor pela Universidade de Salamanca (USAL). Professor na Universidade da Beira Interior (UBI). E-mail: jc@ubi.pt

RECEBIDO EM: 24/03/2012 | ACEITO EM: 02/05/2012 